\title{
Clinical and Economic Burden of Elevated Blood Eosinophils in Patients With and Without Uncontrolled Asthma
}

\author{
Julian Casciano, BS; Jerry Krishnan, MD, PhD; \\ Zenobia Dotiwala, MS; Chenghui Li, PhD; and Shawn X. Sun, PhD
}

\begin{abstract}
BACKGROUND: The European Respiratory Society and American Thoracic Society (ERS/ATS) published guidelines in $\mathbf{2 0 1 4}$ for the evaluation and treatment of asthma. These guidelines draw attention to management of patients with asthma that remains uncontrolled despite therapy. One phenotypic characteristic of therapy-resistant asthma is eosinophil elevation. It is important to better understand the burden of care gaps in this patient subgroup in order to support improved treatment strategies in the future.
\end{abstract}

OBJECTIVE: To quantify the economic burden of asthma patients with and without peripheral blood eosinophil elevation.

METHODS: A retrospective cohort study was conducted using data from patients aged 12 years or older with a diagnosis of asthma using electronic health records of over 2 million patients between 2004-2010. Patients with a diagnosis of chronic obstructive pulmonary disease, Churg Strauss syndrome/Wegener's granulomatosis, eosinophilia, cystic/pulmonary fibrosis, allergic bronchopulmonary aspergillosis, or lung cancer in the 12-month period before the date of asthma diagnosis were excluded. Patients with asthma were followed for 12 months after their initial asthma diagnosis to identify those with controlled versus uncontrolled asthma based on ERS/ATS criteria. Patients with at least 1 peripheral blood eosinophil test result of $\geq 400$ cells $/ \mu \mathrm{L}$ were classified as those with elevated eosinophils. Total annual paid-claim cost was compared by eosinophil levels within the controlled and uncontrolled asthma subgroups. Costs were adjusted to 2015 U.S. dollars. Patients were stratified by control level, and generalized linear modeling regressions were used to assess the magnitude of increase in cost of the elevated eosinophil group.

RESULTS: A total of 2,701 patients were included in the study, of which $17 \%$ had uncontrolled asthma and $21 \%$ had elevated eosinophils. The mean total annual cost of patients with uncontrolled asthma was more than 2 times the cost of those with controlled asthma (\$18,341 vs. $\$ 8,670, P<0.001)$. Patients with uncontrolled asthma in the elevated eosinophil group had almost double the total cost $(\$ 28,644$ vs. $\$ 14,188$, $P=0.008$ ) compared with those with blood eosinophil levels in a normal range. Similarly, patients classified as those with controlled asthma in the elevated eosinophil group had almost twice the average costs as those without elevated eosinophils $(\$ 14,754$ vs. $\$ 7,203, P<0.001)$. Uncontrolled asthma with elevated eosinophils had 4 times greater hospital admissions and over 4 times higher total costs than controlled asthma without elevated eosinophils. Among patients with uncontrolled asthma, patients with elevated eosinophils had a $53 \%$ increase in mean cost ( $\$ 17,723$ vs. $\$ 11,581, P<0.001)$ compared with patients without elevated eosinophils. Among patients with controlled asthma, patients with elevated eosinophils had a $62 \%$ increase in mean cost $(\$ 8,897$ vs. $\$ 5,486, P<0.001)$ compared with patients without elevated eosinophils.

CONCLUSIONS: Elevated peripheral blood eosinophil level is associated with higher cost irrespective of disease control status.

J Manag Care Spec Pharm. 2017;23(1):85-91

Copyright $\odot 2017$, Academy of Managed Care Pharmacy. All rights reserved.

\section{What is already known about this subject}

Guidelines from the European Respiratory Society and American Thoracic Society describe asthma as a heterogeneous condition that may include different phenotypes identified on the basis of various biomarkers, including peripheral eosinophil counts.

Eosinophil elevation is known to be associated with severe asthma and asthma exacerbations.

\section{What this study adds}

Patients with elevated peripheral eosinophil counts incur greater health care expenditures compared with those with lower eosinophil counts.

The association of eosinophil elevation with greater health care burden stands irrespective of asthma control status.

T he U.S. asthma population poses a significant burden to the health care system. In 2011, 18.8 million adults and 7.1 million children were reported to have asthma in the United States. In 2010, 439,000 hospitalizations with a primary diagnosis of asthma were recorded. ${ }^{1}$ The cost of managing uncontrolled asthma has been estimated at more than double that of controlled asthma. ${ }^{2}$

An updated definition of severe asthma, incorporating medication use and control level, has been published in revised evaluation and treatment guidelines by the European Respiratory Society and American Thoracic Society (ERS/ATS). ${ }^{3}$ With its focus on improved management of treatment-resistant, poorly controlled patient segments, the ERS/ATS guidelines align with population health management research, which attributes a sizeable portion of all cost in asthma to just $10 \%$ of patients. ${ }^{4}$ In providing the most recent recommendations for asthma management to date, ERS/ATS promulgates attention to costly unmanaged patient subsets by phenotype, including those characterized using eosinophil biomarkers. Zeiger et al. (2014) link elevated peripheral blood eosinophils ( $\geq 400$ cells $/ \mu \mathrm{L})$ to exacerbations. ${ }^{5}$ They found that the elevated eosinophil count was a risk factor for asthma exacerbations (adjusted rate ratio $=1.31,95 \%$ confidence interval $[\mathrm{CI}]=1.07-1.60 ; P=0.009)$. Other published studies have correlated eosinophil elevation with asthma exacerbations. ${ }^{6,7}$ The objective of this study was to estimate and compare the costs of health care resource use for 
Patients with asthma (ICD-9-CM code 493.xx between 2004-2010) with 1 year of continuous health plan enrollment following diagnosis

$$
\mathrm{N}=7,795
$$

Patients aged $\geq 12$ years

$n=5,529$

Patients without confounding disease states of COPD, emphysema, Churg Strauss syndrome, Wegener's granulomatosis, eosinophilia, pulmonary fibrosis, allergic bronchopulmonary aspergillosis, and lung cancer in the 12-month period before the diagnosis date

$$
n=4,767
$$

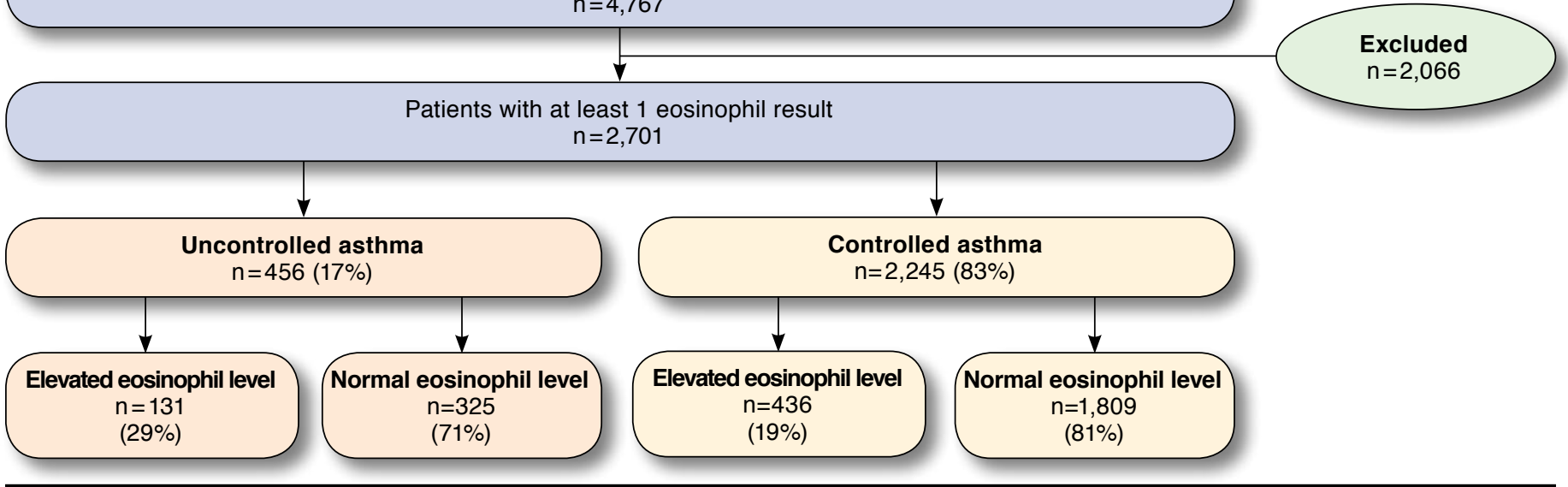

COPD = chronic obstructive pulmonary disease; ICD-9-CM = International Classification of Diseases, Ninth Revision, Clinical Modification.

controlled and uncontrolled asthma patients with and without elevated peripheral blood eosinophil levels.

\section{Methods}

\section{Study Design and Data Source}

A retrospective study of patients with a primary or secondary diagnosis of asthma was conducted using data between January 2004 and July 2010 from EMRClaimst, an integrated health services database of patients located in the Midwest region of the United States. The database includes administrative insurance claims from a managed care plan of approximately 675,000 lives linked to an overlapping health care provider database of electronic medical records (EMR) data including laboratory values and provider billing files. Additionally, this database provides the ability to extract specific physician notes, disease severity, and certain functional test results through access to patient medical charts.

\section{Study Population and Study Measures}

Included in the study were patients aged at least 12 years, with a diagnosis of asthma (International Classification of
Diseases, Ninth Revision, Clinical Modification [ICD-9-CM] code 493.xx) between January 2004 and July 2010, and who were continuously enrolled in their health insurance plans for a period of 12 months following asthma diagnosis. Criteria consistent with published drug registration studies among eosinophilic asthma patients were used to exclude individuals with a diagnosis of chronic obstructive pulmonary disease (ICD-9-CM codes 491.xx-492.xx and 494.xx-496.xx); Churg Strauss syndrome/Wegener's granulomatosis (446.4); eosinophilia (288.3), cystic/pulmonary fibrosis (277.x, 515, and 516.31); allergic bronchopulmonary aspergillosis (518.6); and lung cancer (162.x) in the 12-month period before the date of asthma diagnosis. ${ }^{8}$ The date of asthma diagnosis was defined as the index date; the 12-month period before was defined as the pre-index period. Patients were followed for up to 12 months (post-index period) after the index date to identify individuals with uncontrolled asthma. Patients were identified as those with uncontrolled asthma if, during the same 12-month period, any of the following ERS/ATS criteria were satisfied: 


\begin{tabular}{|c|c|c|c|c|c|}
\hline \multirow[t]{2}{*}{ Demographics/Comorbidities } & \multicolumn{2}{|c|}{ Uncontrolled } & \multicolumn{2}{|c|}{ Controlled } & \multirow{2}{*}{$\begin{array}{c}P \\
\text { Value } \\
\end{array}$} \\
\hline & 456 & $\%$ & 2,245 & $\%$ & \\
\hline \multicolumn{6}{|l|}{ Gender } \\
\hline Female & 326 & 71 & 1,564 & 70 & 0.438 \\
\hline Male & 130 & 29 & 681 & 30 & \\
\hline \multicolumn{6}{|l|}{ Age } \\
\hline $12-18$ years & 18 & 4 & 319 & 14 & \multirow{5}{*}{$<0.001$} \\
\hline $18-35$ years & 70 & 15 & 460 & 20 & \\
\hline $36-45$ years & 76 & 17 & 397 & 18 & \\
\hline $46-65$ years & 197 & 43 & 727 & 32 & \\
\hline$\geq 65$ years & 95 & 21 & 342 & 15 & \\
\hline Mean age (SD) & 50 & $(16.67)$ & 44 & $(19.41)$ & $<0.001$ \\
\hline \multicolumn{6}{|l|}{ Race } \\
\hline White & 219 & 48 & 977 & 44 & \multirow{4}{*}{0.019} \\
\hline African American & 50 & 11 & 375 & 17 & \\
\hline Hispanic & 114 & 25 & 553 & 25 & \\
\hline Other & 73 & 16 & 340 & 15 & \\
\hline \multicolumn{6}{|l|}{ Comorbidities } \\
\hline Diabetes & 66 & 14 & 240 & 11 & 0.020 \\
\hline $\mathrm{CHF}$ & 17 & 4 & 74 & 3 & 0.641 \\
\hline Malignant lymphoma/leukemia & 16 & 4 & 64 & 3 & 0.449 \\
\hline Rheumatic disease & 5 & 1 & 39 & 2 & 0.324 \\
\hline Chronic pulmonary disease & 9 & 2 & 33 & 1 & 0.428 \\
\hline \multicolumn{6}{|l|}{ CCI score } \\
\hline 0 & 341 & 75 & 1,821 & 81 & \multirow{3}{*}{0.004} \\
\hline $1-3$ & 103 & 23 & 393 & 18 & \\
\hline$\geq 4$ & 12 & 3 & 31 & 1 & \\
\hline Mean CCI score (SD) & 0.46 & $(1.05)$ & 0.33 & $(0.86)$ & 0.015 \\
\hline
\end{tabular}

1. Two or more courses of systemic corticosteroid (SCS) of more than 3 days each, identified based on the presence of 2 or more pharmacy claims with more than 3 days of supply.

2. Serious exacerbations defined as the following:

- At least 1 hospitalization with a primary discharge diagnosis of asthma, or

- Admission to an intensive care unit for any cause, or

- Use of mechanical ventilation.

3. Airflow limitation: after appropriate bronchodilator withhold FEV1, $80 \%$ predicted (in the face of reduced FEV1/FVC defined as less than the lower limit of normal), identified through manual digital chart search and abstraction.

Patients not meeting the uncontrolled asthma definition within 12 months of asthma diagnosis were defined as those with controlled asthma. Patients presenting with uncontrolled asthma symptoms were also classified as severe asthma patients based on use of either (a) high-dose inhaled corticosteroids (defined by ERS/ATS guidelines ${ }^{3}$ ) with long-acting beta agonists or leukotriene modifier or theophylline or (b)
SCS for greater than 50\% of the post-index period (calculated based on days supply during the 12-month period). Patients were required to have at least 1 eosinophil test result during the study period (pre-index and post-index period). Previous literature has studied the association of elevated eosinophils, asthma severity, and exacerbations. Researchers have classified eosinophils as elevated at $\geq 400$ cells $/ \mu \mathrm{L},{ }^{5-7}$ at $\geq 300$ cells $/ \mu \mathrm{L},{ }^{9}$ and at $\geq 140$ cells $/ \mu \mathrm{L} .{ }^{10}$ Price et al. (2015) studied the association of elevated eosinophils at different cut-off levels (including $\geq 300$ cells $/ \mu \mathrm{L}$ and $\geq 200$ cells $/ \mu \mathrm{L}$ ) with exacerbations and found that association diminishes to a large extent at thresholds below $\geq 400$ cells $/ \mu \mathrm{L} .{ }^{7}$ Given similar findings that we have previously reported, ${ }^{11}$ in this study, we define elevated eosinophils based on at least 1 test result observation of $\geq 400$ cells/ $\mu \mathrm{L}$ and normal eosinophils as all test results $<400$ cells $/ \mu \mathrm{L}$.

Demographics such as age, gender, and race were recorded at asthma date, and comorbidities included in the Charlson Comorbidity Index $(\mathrm{CCI})^{12}$ were identified during the 12 -month period before asthma diagnosis. The total medical (admissions, emergency room visits, and outpatient visits) and prescription drug cost during the 12-month period following asthma diagnosis was recorded for each patient. Cost for each of these resource use measures was estimated using either insurance claims or provider billed amounts (charges) in the absence of third-party claims and converted to cost based on cost-tocharge ratio factor (0.33) derived through an analysis of 13,000 asthma-patient encounters at over 95 hospitals in the Midwest as previously described. ${ }^{13}$ Costs were adjusted to reflect 2015 U.S. dollars. ${ }^{14}$

\section{Data Analysis}

Descriptive Analysis. Percentage of patients with uncontrolled and controlled asthma, as well as elevated eosinophils and normal eosinophil levels were reported. Demographic distributions were calculated for uncontrolled and controlled asthma patients. Total resource use and cost during the 12-month period following asthma diagnosis was calculated and compared by eosinophil level separately for uncontrolled and controlled asthma patients. Mean values were compared statistically using t-tests, and categorical outcomes were assessed using chi-square testing or Fisher exact tests.

Multivariate Analysis. Patients were stratified based on control status, and generalized linear modeling (GLM) regressions with gamma distribution and log link were used to assess the association of eosinophil elevation on mean cost. Covariates used were gender, age, and CCI score.

All data analyses were conducted using SAS software, version 9.3 (SAS Institute, Cary, NC). 
FIGURE 2 Mean (SD) Total Annual Cost Comparison Between Groups with Elevated and Normal Eosinophil Levels by Control Status, Severity, and Overall

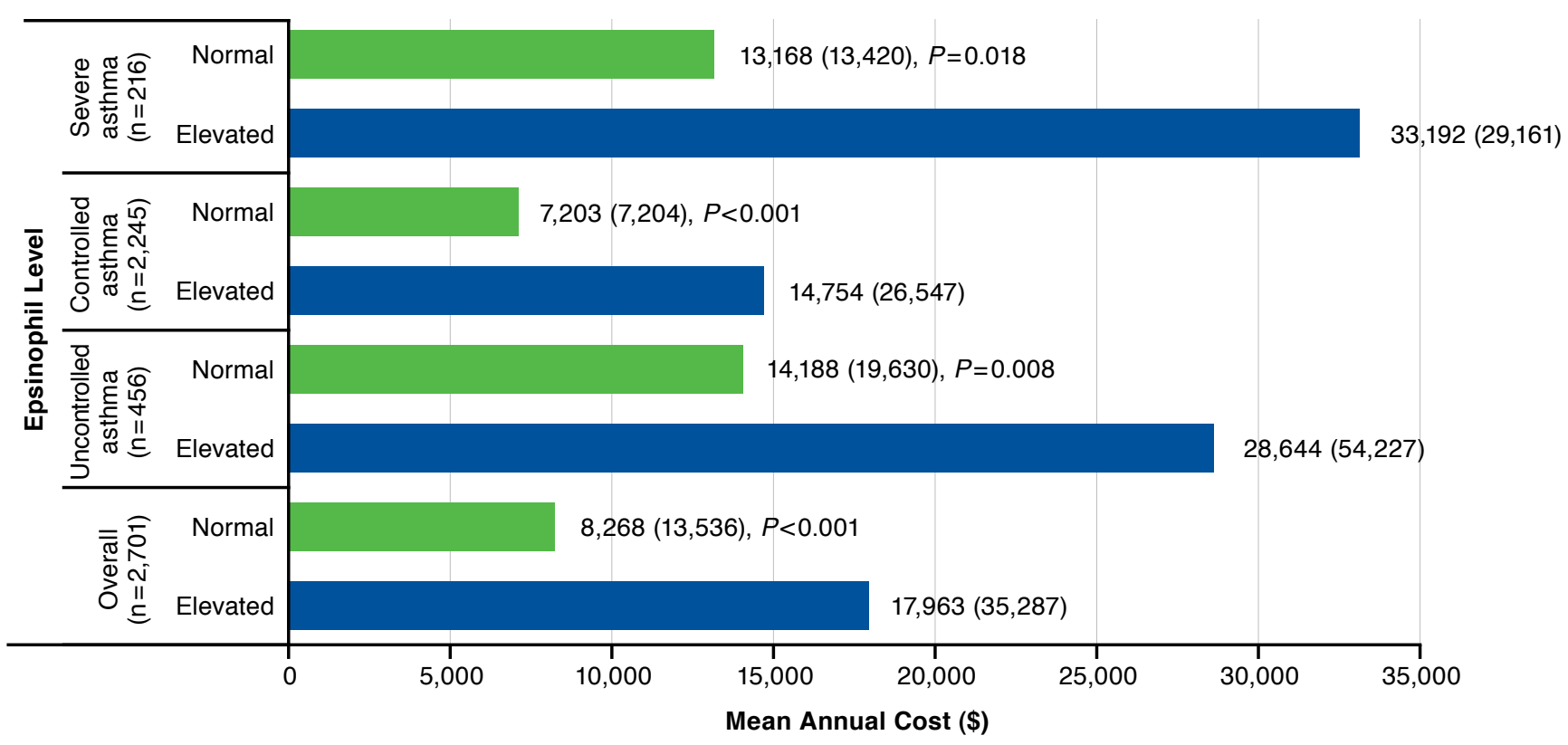

$S D=$ standard deviation

\section{Results}

\section{Study Sample, Demographics, and Distribution}

Included in this study were 2,701 patients, of which $17 \%$ $(\mathrm{n}=456)$ were shown to have uncontrolled asthma (Figure 1). Demographics and comorbidities are shown in Table 1. There was a greater proportion of females overall, but there was no significant difference in gender distribution between controlled and uncontrolled asthma patients $(P=0.438)$. The mean patient age for the uncontrolled asthma group was significantly greater than those with controlled asthma (50 years vs. 44 years, $P<0.001$ ). Uncontrolled asthma patients had a significantly greater mean CCI score compared with controlled asthma (0.46 vs. $0.33, P=0.015)$. Twenty-nine percent $(n=131)$ of patients with uncontrolled asthma and 19\% ( $\mathrm{n}=436)$ of patients with controlled asthma had elevated eosinophil levels. About $8 \%$ of the study sample was classified as having severe asthma ( $\mathrm{n}=216)$.

\section{Resource Use and Economic Comparisons}

The mean (standard deviation [SD]) total annual cost of patients with uncontrolled asthma was 2.1 times greater than the group with controlled asthma $(\$ 18,341[33,907]$ vs. $\$ 8,670$ $[16,047], P<0.001)$. The mean (SD) total annual cost was significantly greater for the group with elevated eosinophils compared with normal eosinophils $(\$ 17,963$ [35,287] vs. $\$ 8,268$ [13,535], $P<0.001$ ) overall (Figure 2). Restricting the sample to those with uncontrolled asthma, patients with elevated eosinophils had more than double the total cost on average compared to patients with normal eosinophils $(\$ 28,644[54,227]$ vs. $\$ 14,188[19,630], P=0.008)$. For controlled asthma, those with elevated eosinophil levels also had significantly greater mean cost $(\$ 14,754[26,547]$ vs. $\$ 7,203$ [11,874], $P<0.001$ ), as did patients in the severe asthma group $(\$ 33,192[29,161]$ vs. $\$ 13,168[13,420], P=0.018)$.

As shown in Figure 3, patients with elevated eosinophils had greater mean number of admissions annually, compared with those with normal eosinophils throughout the different groups (overall mean [SD]: 0.5 [1.2] vs. 0.2 [0.6], $P<0.001$; uncontrolled asthma mean [SD]: 0.8 [1.5] vs. 0.4 [0.9], $P=0.008$; controlled asthma mean [SD]: 0.5 [1.1] vs. 0.2 [0.5], $P<0.001$; and severe asthma mean [SD]: 0.8 [1.6] vs. 0.3 [0.6], $\mathrm{P}=0.020$ ).

GLM regression showed that eosinophil elevation was associated with $62 \%$ greater cost $(P<0.001)$ compared with patients with normal eosinophils (Table 2) after controlling for asthma-control status and demographics. Each unit increase in CCI score and age was associated with a 35\% and 3\% increase in cost, respectively $(P<0.001$ each). Within the uncontrolled asthma subgroup, elevated eosinophil patients were associated 


\section{FIGURE 3 Mean (SD) Total Annual Admissions Comparison Between Groups with Elevated and Normal} Eosinophil Levels by Control Status, Severity, and Overall

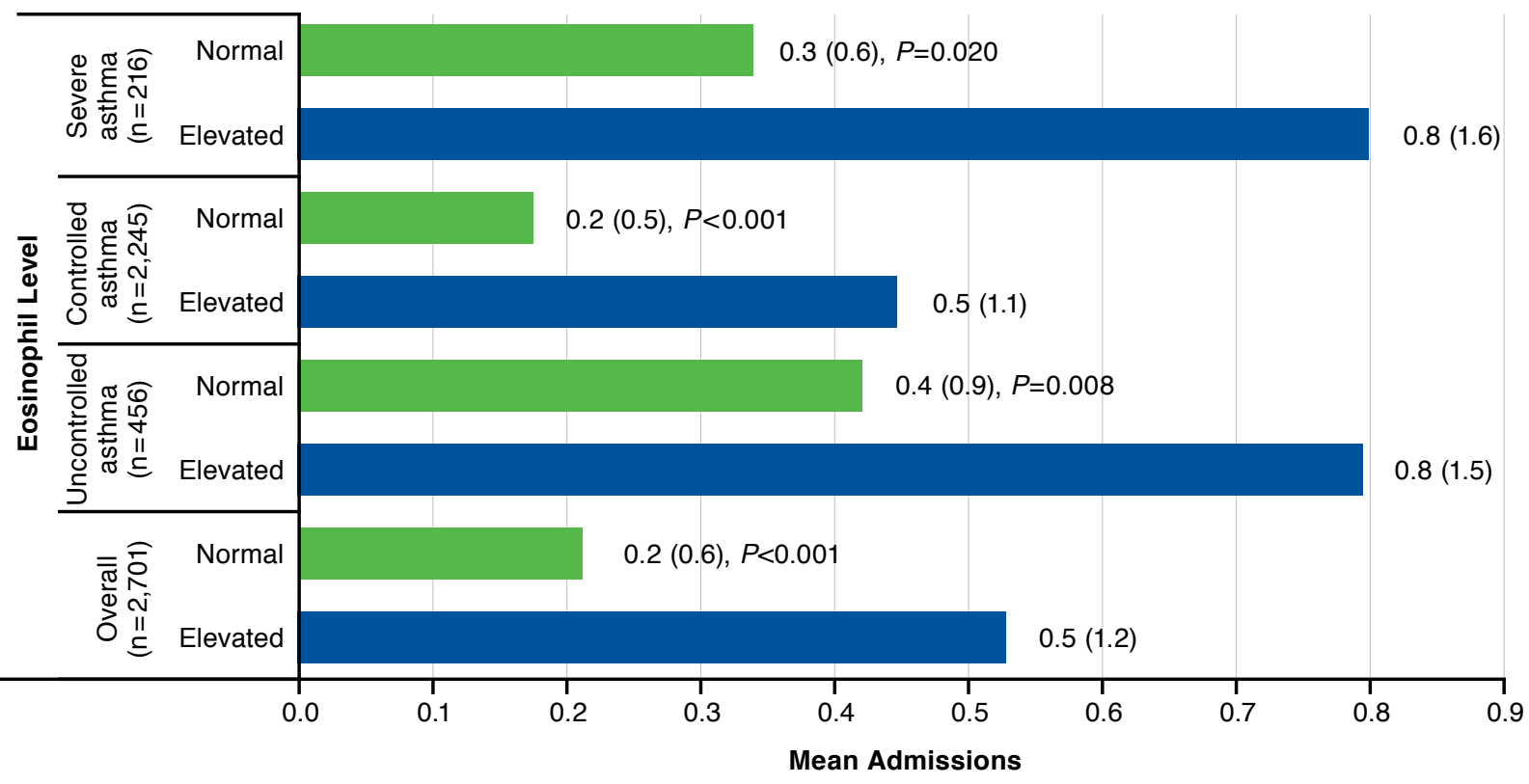

SD = standard deviation

with a 53\% increase in mean cost compared to the normal eosinophil group (cost ratio $[\mathrm{CR}]=1.53$, 95\% CI $=1.22-1.93$ ). Similarly, elevated eosinophil level was associated with a $62 \%$ increase in mean cost within the controlled asthma subgroup $(\mathrm{CR}=1.62,95 \% \mathrm{CI}=1.45-1.82)$.

\section{Discussion}

The economic and clinical burden of uncontrolled asthma has been extensively studied with various definitions using questionnaires, productivity assessments, and the Global Initiative for Asthma guidelines. ${ }^{15}$ The literature is consistent in reporting a greater burden among this difficult-to-manage subgroup of asthma patients, and our findings build upon this body of work. 2,16,17 We found uncontrolled asthma to be a significant driver of total health care cost. In fact, Sullivan et al. (2007) reported that costs for uncontrolled asthma $(\$ 14,212)$ were more than double that of controlled asthma $(\$ 6,452)$, which is precisely in line with our study results. ${ }^{2}$

Setting this research apart, our lens is enhanced by new symptom control definitions offered in the ERS/ATS report. The formation of an expert group by the National Institute of Health (NIH) to classify key biological outcome measures for federally sponsored asthma research demonstrates a movement to better align patient phenotype with choice of therapeutic intervention. The importance of blood eosinophil measurement is evidenced by its recommendation as a supplemental biomarker by the NIH asthma report. ${ }^{17}$

What remains underreported in the literature is the large increase in cost corresponding to eosinophil elevation. ${ }^{3}$ We found that within our group of patients with uncontrolled asthma, a population already incurring greater cost, a comparative subgroup of patients with eosinophil elevation were associated with significantly greater expenditures. This relationship held true within the controlled asthma subgroup as well. Furthermore, this subgroup of elevated eosinophil patients registers almost twice the cost of those without elevation.

Eosinophil elevation leads to greater costs, irrespective of control classification, thus highlighting that blood eosinophil level is an important biomarker to consider during asthma management, especially for patients with difficult-to-control asthma. The need to focus on better management of uncontrolled asthma is well documented in the literature. However, this study describes the gap in clinical management of the elevated eosinophil subgroup.

\section{Limitations}

This study has some limitations that should be acknowledged. Asthma control status may have been misclassified because of lack of patient symptom data. Patient-reported outcomes were not included in our definition of asthma control, and we did not 
TABLE 2 Factors Associated with Total Annual Cost

\begin{tabular}{|c|c|c|c|c|}
\hline Independent Variables & Exponentiated Estimate & LCL & $\mathrm{UCL}$ & $P$ Value \\
\hline \multicolumn{5}{|l|}{ Overall $(n=2,701)$} \\
\hline Elevated eosinophils (vs. normal) & 1.62 & 1.46 & 1.80 & $<0.001$ \\
\hline Uncontrolled asthma (vs. controlled) & 1.71 & 1.54 & 1.91 & $<0.001$ \\
\hline Female (vs. male) & 1.07 & 0.97 & 1.17 & 0.158 \\
\hline Age & 1.03 & 1.02 & 1.03 & $<0.001$ \\
\hline African American (vs. Caucasian) & 0.96 & 0.85 & 1.08 & 0.520 \\
\hline Hispanic (vs. Caucasian) & 0.95 & 0.85 & 1.05 & 0.310 \\
\hline Other (vs. Caucasian) & 0.92 & 0.82 & 1.04 & 0.182 \\
\hline CCI score & 1.35 & 1.28 & 1.42 & $<0.001$ \\
\hline \multicolumn{5}{|c|}{ Uncontrolled asthma patients only $(n=456)$} \\
\hline Elevated eosinophils (vs. normal) & 1.53 & 1.22 & 1.93 & $<0.001$ \\
\hline Female (vs. male) & 1.03 & 0.82 & 1.31 & 0.773 \\
\hline Age & 1.01 & 1.01 & 1.02 & $<0.001$ \\
\hline African American (vs. Caucasian) & 0.86 & 0.61 & 1.21 & 0.397 \\
\hline Hispanic (vs. Caucasian) & 0.93 & 0.72 & 1.19 & 0.558 \\
\hline Other (vs. Caucasian) & 0.94 & 0.70 & 1.27 & 0.702 \\
\hline CCI score & 1.55 & 1.38 & 1.74 & $<0.001$ \\
\hline \multicolumn{5}{|c|}{ Controlled asthma patients only $(n=2,245)$} \\
\hline Elevated eosinophils (vs. normal) & 1.62 & 1.45 & 1.82 & $<0.001$ \\
\hline Female (vs. male) & 1.08 & 0.98 & 1.20 & 0.107 \\
\hline Age & 1.03 & 1.03 & 1.03 & $<0.001$ \\
\hline African American (vs. Caucasian) & 0.98 & 0.86 & 1.11 & 0.750 \\
\hline Hispanic (vs. Caucasian) & 0.95 & 0.85 & 1.06 & 0.375 \\
\hline Other (vs. Caucasian) & 0.91 & 0.80 & 1.04 & 0.157 \\
\hline CCI score & 1.28 & 1.21 & 1.36 & $<0.001$ \\
\hline
\end{tabular}

identify control status based on poor symptom control, using the Asthma Control Questionnaire as noted in the ERS/ATS guidelines. The influence of other potentially important patient-related factors (e.g., smoking status) was not considered. The reason for eosinophil elevation was not accounted for in this study and could be present, which may lead to an overestimation of patients with eosinophil elevation because of underlying asthma. Almost half of the patients identified initially with asthma $(2,066$ of 4,767$)$ did not have an eosinophil level. Patients who have an eosinophil level may be different in clinical presentation or severity (more severe) compared with patients who never receive an eosinophil test; therefore, our results cannot be generalized to the entire asthma population. This study used data from 7 states in the midwestern United States so may not be nationally representative. Additionally, the GLM only controlled for observable factors present in the data.

\section{Conclusions}

Elevated eosinophil level was associated with additional health care expenditure among patients with uncontrolled and controlled asthma.

\section{Authors}

JULIAN CASCIANO, BS, and ZENOBIA DOTIWALA, MS, eMAX Health, White Plains, New York. JERRY KRISHNAN, MD, PhD, University of Illinois Hospital and Health Sciences System, Chicago; CHENGHUI LI, PhD, University of Arkansas for Medical Sciences, Little Rock; and SHAWN X. SUN, PhD, Teva Pharmaceuticals, Frazer, Pennsylvania.

AUTHOR CORRESPONDENCE: Zenobia Dotiwala, eMAX Health, 445 Hamilton Ave., Ste. 1102, White Plains, NY 10601.

Tel.: 914.304.8128; Fax: 914.206.4959;

E-mail: zenobiadotiwala@emaxhealth.net.

\section{DISCLOSURES}

This study was funded by Teva Pharmaceuticals. Dotiwala and Casciano report consulting and writing fees from Teva Pharmaceuticals for work on this study. Sun is an employee and stockholder of Teva Pharmaceuticals. Li reports consulting fees from eMAX Health.

All authors contributed to study design. Dotiwala took the lead in data collection, along with the other authors, and data interpretation was performed primarily by Krishnan, Sun, and Li, along with Casciano and Dotiwala. The manuscript was written by Casciano, Dotiwala, and Li, along with Sun and Krishnan, and revised by Casciano, Dotiwala, Sun, and Li, with assistance from Krishnan. 


\section{REFERENCES}

1. American Lung Association, Epidemiology \& Statistics Unit, Research and Program Services. Trends in asthma morbidity and mortality. September 2012. Available at: http://www.lung.org/assets/documents/research/asthmatrend-report.pdf. Accessed November 15, 2016.

2. Sullivan S, Rasouliyan L, Russo PA, Kamath T, Chipps BE; TENOR Study Group. Extent, patterns, and burden of uncontrolled disease in severe or difficult-to-treat asthma. Allergy. 2007;62(2):126-33.

3. Chung KF, Wenzel SE, Brozek JL, et al. International ERS/ATS guidelines on definition, evaluation and treatment of severe asthma. Eur Respir J. 2014;43(2):343-73

4. Moore WC, Bleecker ER, Curran-Everett D, et al. Characterization of the severe asthma phenotype by the National Heart, Lung, and Blood Institute's Severe Asthma Research Program. J Allergy Clin Immunol. 2007;119(2):405-13.

5. Zeiger R, Schatz M, Li Q, et al. High blood eosinophil count is a risk factor for future asthma exacerbations in adult persistent asthma. J Allergy Clin Immunol. 2014;2(6):741-50.

6. Tran T, Khatry D, Ke X, Ward C, Gossage D. High blood eosinophil count is associated with more frequent asthma attacks in asthma patients. Tran Ann Allergy Asthma Immunol. 2014;113(1):19-24.

7. Price D, Rigazio A, Campbell J, et al. Blood eosinophil count and prospective annual asthma disease burden: a UK cohort study. Lancet Respir Med. 2015;3(11):849-58.

8. Castro M, Zangrilli J, Wechsler M, et al. Reslizumab for inadequately controlled asthma with elevated blood eosinophil counts: results from two multicentre, parallel, double-blind, randomised, placebo-controlled, phase 3 trials. Lancet Respir Med. 2015;3(5):355-66.

9. Pavord ID, Korn S, Howarth P, et al. Mepolizumab for severe eosinophilic asthma (DREAM): a multicentre, double-blind, placebo-controlled trial. Lancet. 2012;380(9842):651-59.
10. Corren J, Lemanske R, Hanania N, et al. Lebrikizumab treatment in adults with asthma. N Engl J Med. 2011;365(12):1088-98.

11. Casciano J, Krishnan J, Buatti Small M, et al. The association of blood eosinophil levels and asthma severity. Poster presented at: American College of Allergy, Asthma and Immunology Conference; November 2013; Baltimore, MD.

12. Charlson ME, Pompei P, Ales KL, MacKenzie CR. A new method of classifying prognostic comorbidity in longitudinal studies: development and validation. J Chronic Dis. 1987;40(5):373-83.

13. Casciano J, Krishnan J, Buatti Small M, et al. Cost-consequence of elevated versus normal EOS among patients that followed medication use recommended by guidelines for severe asthma. Poster presented at: ISPOR 20th Annual International Meeting; May 16-20, 2015; Philadelphia, PA. Available at: http://www.ispor.org/research_pdfs/49/pdffiles/PRS32.pdf. Accessed November 15, 2016.

14. U.S. Department of Labor, Bureau of Labor Statistics. CPI inflation calculator. Available at: http://data.bls.gov/cgi-bin/cpicalc.pl. Accessed November 15, 2016.

15. National Heart, Lung, and Blood Institute, National Asthma Education and Prevention Program. Expert panel report 3: guidelines for the diagnosis and management of asthma. NIH Publication No. 07-4051. Revised August 28, 2007. Available at: http://www.nhlbi.nih.gov/files/docs/guidelines/asthgdln.pdf. Accessed November 15, 2016.

16. Peters S, Ferguson G, Deniz Y, Reisner C. Uncontrolled asthma: a review of the prevalence, disease burden and options for treatment. Respir Med. 2006;100(7):1139-51.

17. Dean B, Calimlim B, Kindermann S, Khandker R, Tinkelman D. The impact of uncontrolled asthma on absenteeism and health-related quality of life. J Asthma. 2009;46(9):861-66.

18. Szefler SJ, Wenzel S, Brown R, et al. Asthma outcomes: biomarkers. J Allergy Clin Immunol. 2012;129(3 Suppl): S9-23. 